\title{
Minireview
}

\section{Comparative pharmacology of oral fluoropyrimidines: a focus on pharmacokinetics, pharmacodynamics and pharmacomodulation}

\author{
G Milano*,', J-M Ferrero' and E François' \\ 'Oncopharmocology Unit, Centre Antoine-Lacassagne, 33, Avenue de Valombrose, 06189 Nice Cedex 2, France
}

\begin{abstract}
The main purpose of the present review article was to shed light on the different 5-fluorouracil (5-FU) prodrugs by underlining their respective pharmacological features in terms of metabolic activation, dihydropyrimidine dehydrogenase inhibition, pharmacokinetic profile and biomodulation ability. Oral fluoropyrimidines differ particularly as concerns their pharmacokinetic profile and especially in the delivery of circulating 5-FU. More clinical studies need to be performed incorporating tumour predictive markers during oral fluoropyrimidine-based treatment. The new possibilities are to achieve pharmacomodulation of oral fluoropyrimidines, notably for UFT and capecitabine, that open up the prospect of establishing significant novel treatment protocols based on drug combinations. British Journal of Cancer (2004) 91, 613-617. doi: I0.1038/sj.bjc.6601973 www.bjcancer.com
\end{abstract}

Published online 27 July 2004

(c) 2004 Cancer Research UK

Keywords: oral chemotherapy; UFT; capecitabine

5-Fluorouracil (5-FU) remains one of the most commonly prescribed anticancer drugs with significant activity against cancers of the gastrointestinal tract, head and neck and breast. Repeated intravenous administrations or protracted infusions are heavy for patients. Other oral 5-FU prodrugs, with new pharmacological characteristics, are now emerging in the clinical area in oncology (Diasio, 1999). These 5-FU prodrugs differ markedly in their mode of activation, their pharmacokinetic behaviour, particularly in terms of dihydropyrimidine dehydrogenase (DPD) inhibition, and their pharmacologic modulation. Table 1 describes the 5-FU prodrugs currently at more or less advanced stage of clinical development. Previous reviews on oral fluoropyrimidines have provided extensive description of the different characteristics of these drugs (Diasio, 1999). The purpose of the present review is to propose a transversal pharmacological analysis of these oral fluoropyrimidines. Thus, special attention will be paid to metabolic activation, DPD inhibition, pharmacokinetic profile and biomodulation ability leading to compare oral fluoropyrimidines between them at the light of these latter pharmacological criteria. This information could promote a better understanding of their pharmacological properties as well as enabling optimal clinical use in the framework of the currently approved therapeutic schedules and in the context of future clinical developments.

\section{DPD INHIBITION}

More than $80 \%$ of an administered dose of 5 -FU is eliminated by catabolism through DPD which is the rate-limiting enzyme of pyrimidine catabolism (Grem, 1996). The combination of DPD inhibitors with 5-FU itself or 5-FU prodrugs has several potential

*Correspondence: Mr G Milano; E-mail: gerard.milano@nice.fnclcc.fr Received 16 January 2004; revised 20 April 2004; accepted I I May 2004; published online 27 July 2004 pharmacologic benefits conferred by the inhibition of 5-FU catabolism during gastrointestinal absorption and first pass in the liver. Furthermore, DPD inhibition improves pharmacokinetic behaviour of delivered 5-FU by reducing interpatient variability and by increasing 5-FU half-life. This latter benefit is particularly useful in limiting repeated oral administrations of the drug which is uncomfortable for patients. A large spectrum of DPD inhibitors are used in combination with 5-FU or 5-FU prodrugs. At one extreme, there is eniluracil, which has no cytotoxic activity but which constitutes the most efficacious DPD inhibitor, acting by DPD inactivation through the formation of a covalent bond; this covalent reaction irreversibly inhibits DPD activity at its active site. At the other extreme is UFT including uracil (U) which is a simple competitive inhibitor of DPD activity since it is the natural substrate of DPD. S-1 incorporates another DPD competitor, 5chloro-2,4-dihydroxypyridine which is 200 -fold more potent than $\mathrm{U}$ (Shimasaka et al, 1996). BOF-A2 is an oral prodrug of 5-FU and 3-cyano-2,6-dihydropyrimidine (CNDP). BOF-A2 is rapidly transformed by esterification into two $1: 1$ components, namely, 1ethoxymethyl-5-fluorouracil and CNDP. CNDP is also a competitive inhibitor of DPD with a potency of 2000 times greater than that of U (Tatsumi et al, 1993). Capecitabine does not incorporate a DPD inhibitor and is converted to the cytotoxic moiety 5-FU in target tissue through a series of three metabolic steps (Diasio, 1999). The development of the compound RO0094889 which generates the DPD inhibitor 5-vinyluracil preferentially in tumours by the same enzymes as in capecitabine activation (Hattori et al, 2003) may lead to an interesting combination RO0094889capecitabine to be further explored at the clinical level (Endo et al, 2003).

\section{PHARMACOKINETICS}

Oral fluoropyrimidines differ particularly as concerns their pharmacokinetic profile and especially in the delivery of circulating 5-FU. The DPD inactivator eniluracil is administered with 5-FU 
Table I Oral fluoropyrimidines under clinical evaluation

\begin{tabular}{llll}
\hline Compound & Chemical name & 5-FU prodrug & Effect on DPD \\
\hline $\begin{array}{l}\text { Eniluracil (Glaxo-Wellcome) (Clinical development } \\
\text { currently stopped) }\end{array}$ & 5 -Ethynyluracil & No & Inactivator (complete DPD inhibition) \\
UFT (Orzel ${ }^{\mathbb{R}}$, Bristol-Myers Squibb, contains UFT & Uracil+tegafur & Yes & Inhibitor \\
plus leucovorin) & $\begin{array}{l}5 \text {-Chloro-2, } 4 \\
\text { S-I (Bristol-Myers Squibb) } \\
\text { oxonate }\end{array}$ & Yes & Inhibitor \\
BOF-A2 (Emitefur ${ }^{\mathbb{R}}$, Otsuka America & $\begin{array}{l}\text { I-Ethoxymethyl-5-fluorouracil+3-cyano-2,6 } \\
\text { dihydropyrimidine }\end{array}$ & Yes & Inhibitor \\
Pharmaceutical) & $\begin{array}{l}N^{4} \text {-pentyloxycarbonyl-5'-deoxy-5- } \\
\text { Capecitabine }\left(\text { Xeloda }{ }^{\circledR}, \text { Roche) }\right.\end{array}$ & Yes & No DPD inhibitor \\
\hline
\end{tabular}

in a 10:1 ratio and produces 5-FU directly in the blood compartment. 5-FU pharmacokinetics during multiple oral dosing of eniluracil and 5-FU have been reported by Baker et al (2000). Elimination half-life of 5-FU was around $4.0 \mathrm{~h}$. Between day 2 and day 29 the main pharmacokinetic parameters remained constant, notably for the values of the total body clearance (Baker et al, 2000). Interestingly, Adjei et al (2002) recently reported on a randomised, open-label, cross-over study comparing continuous venous infusion (CVI) of 5-FU to 5-FU/eniluracil combination. The authors noted that individual 5-FU concentrations during CVI were highly variable whereas those after 5-FU/eniluracil were more reproducible, this lesser variability in 5-FU concentrations following 5-FU/eniluracil being attributable to the inhibition of DPD.

The pharmacokinetics of 5-FU following the administration of S- 1 at a standard dose of $80 \mathrm{mg} \mathrm{m}^{-2}$ day $^{-1}$ were first reported by Hirata et al (1999). This relative stability in the pharmacokinetics of 5-FU during S-1 treatment was not in agreement with the recent data by Peters et al (2003), who reported an increase in 5-FU and uracil plasma concentration during repeated daily administration of S-1. Thus, more data are needed about the time-stability of the pharmacokinetics of S-1 during repeated administration. Elimination half-life of 5-FU during S-1 treatment was reported to be in the range of 1.9-2.9h (Hirata et al, 1999). This slowed 5-FU elimination as compared to 5-FU given alone reflects the presence of the DPD inhibitor in S-1. The fate of 5-FU following the administration of BOF-A2 was examined during a phase I doseescalating trial $\left(200 \mathrm{mg} \mathrm{m}^{-2}\right.$ day $^{-1}$ b.i.d. to $300 \mathrm{mg} \mathrm{m}^{-2}$ day $^{-1}$ t.i.d., Nemunaitis et al, 2000). The mean steady-state concentration of plasma 5-FU was in the range of $30-100 \mathrm{ng} \mathrm{ml}^{-1}$. A lack of variation of 5-FU through levels within a day at steady state could be explained by the suppression of circadian variations in 5-FU concentrations due to the DPD inhibition (Petit et al, 1988).

Average plasma concentrations of 5-FU generated from FT in patients treated with oral UFT were comparable to those observed in CVI-treated patients (Ho et al, 1998). Owing to the presence of uracil the 5-FU elimination half-life was markedly higher during UFT treatment $(7.2 \pm 3.9 \mathrm{~h}$ on day 5$)$ as compared to CVI $(0.19 \mathrm{~h})$. Muggia et al (1996) examined 5-FU AUC levels as a function of the timed dose of UFT, $300 \mathrm{mg} \mathrm{m}^{-2}(0800 v s 1800 \mathrm{~h})$. Although not significant, higher 5-FU blood exposures (AUC) were observed in the afternoon dose as compared to the morning. The fact that patients were not their own controls limits the conclusions of the latter study but, from a practical point of view, it would be particularly important to know the magnitude of the difference in 5-FU availability between morning and afternoon administration of UFT. A study was undertaken to address the possible pharmacokinetic influence of concurrent oral administration of UFT and LV (Meropol et al, 1999). When LV was coadministered with UFT, there was no significant impact on the fates of FT, uracil and, particularly, on 5-FU AUC values. Urien et al (2003) recently examined intrapatient variations in the UFT pharmacokinetics on the first cycle of treatment. A steady state was attained for FT and 5 -FU at least on day 8 and there was no further cumulative increase in the AUC of these compounds after 1 week of treatment. In contrast, Ho et al (2000) noted that repeated treatment with UFT led to more or less marked cumulative increase in most of the clinically relevant pharmacokinetic parameters. Thus, larger clinical pharmacokinetic studies are still needed to examine more thoroughly the evolution of 5-FU concentration profile during repeated UFT treatment. It would be of potential interest to check whether the 7-day rest period after the 28-day treatment course allows or not 5-FU AUC to return to values close to those observed at the start of treatment.

The clinical pharmacokinetics of capecitabine have been recently reviewed by Reigner et al (2001). The preferential delivery of 5-FU into the tissues through the intermediary of thymidine phosphorylase (TP) is responsible for its much lower presence (approximately 10 times lower) in plasma than its prodrugs capecitabine, $5^{\prime} \mathrm{DFCR}, 5^{\prime} \mathrm{DFUR}$ or its catabolites FUH2 and FBAL (Reigner et al, 2001). Reigner et al (2001) noted that the AUC of capecitabine, $5^{\prime}$-DFCR and $5^{\prime}$-DFUR did not accumulate in plasma after long-term administration. We recently conducted a phase I and pharmacokinetic study of the association of capecitabinecisplatin in head and neck cancer patients (Pivot et al, 2003) and found that there was a significant but moderate increase in postdose (capecitabine, $1000 \mathrm{mg} \mathrm{m}^{-2}$ ) AUC values for $5^{\prime}$ DFUR and 5 -FU between day 2 and day 15. Interestingly, these pharmacokinetic changes were reversible after the intercycle resting period. However, more knowledge is still needed about the stability of the pharmacokinetic parameters of capecitabine and UFT during their repeated administration.

Perhaps more relevant than plasma pharmacokinetics are data regarding the fate of anticancer drugs in tumours. In colorectal patients treated by UFT, Sadahiro et al (2001) examined the respective concentrations of 5-FU in serum, tumour and normal mucosa at various intervals after the final dose of UFT. While the serum 5-FU concentration decreased to very low levels by $24 \mathrm{~h}$ following the UFT dose, the intratumour concentration of 5-FU had been lowered to only about half, and drug levels in normal mucosa were maintained at least $48 \mathrm{~h}$ after the final dose. Concentrations of 5-FU in the normal mucosa were approximately one-third of those measured in the tumour. Similar observations had been previously reported in head and neck tumours for patients pretreated for 1 week with UFT before surgery (Tachibana et al, 1987). Higher concentrations of 5-FU in tumours were reported for colorectal cancer patients treated by capecitabine (Schüller et al, 2000). Patients received $1225 \mathrm{mg} \mathrm{m}^{-2}$ twice daily for 5-7 days prior to surgery. The ratio of 5-FU concentrations in tumour to adjacent healthy tissue averaged 3.2 while the mean 
tissue/plasma 5-FU concentration ratio exceeded 20 for colorectal tumour and ranged from 8 to 10 for other tissues. Data in breast cancer patients treated by capecitabine are more contrasted with no significant differences for the levels of capecitabine and its metabolites between healthy and malignant tissue (Mader et al, 2003).

The influence of various covariables including gender, biological functions, food intake and coadministration of other drugs has been examined for the pharmacokinetics of oral fluoropyrimidines. Pharmacokinetic changes due to food have been particularly well studied for UFT and capecitabine. For this latter drug it was shown by Reigner et al (1998) that food had marked effects on the AUC of capecitabine only (50\% reduction) while the impact on metabolites in plasma was minor. Consequently, it was recommended that capecitabine be administered with food. In contrast it was found for UFT that food significantly decreased the maximal plasma concentrations and AUC values of uracil and 5-FU (Damle et al, 2001). These observations led to the conclusion not to administer UFT simultaneously with food. The administration of food with oral 5-FU and eniluracil has also been considered (Shepard et al, 2002); the pharmacokinetic data show a slowed absorption of 5-FU and decreased 5-FU $C_{\max }$ but without significant effect on AUC.

The impact of gastrectomy has not been sufficiently explored for oral fluoropyrimidines. Hirata et al (1999) compared the pharmacokinetic parameters of 5-FU following administration of S-1 among patients who underwent gastrectomy and those who did not. They found that the influence of gastrectomy was minor. Few studies have considered the possible influence of deteriorated renal or hepatic functions on the pharmacokinetics of oral fluoropyrimidines and more investigations at this level must be encouraged. This lack of information is surprising as hepatic dysfunction is relatively common in patients with cancer of the breast, colon or rectum, which are tumoural localisations with a high incidence of liver metastases and particularly concerned by the current development of oral fluoropyrimidines. Twelves et al (1999) examined the impact of hepatic dysfunction due to liver metastases on the pharmacokinetics of capecitabine and its metabolites. Counterintuitively, maximal plasma concentrations and AUC values of the main metabolites of capecitabine were increased in patients with liver dysfunction. These differences, however, did not reach statistical significance and the authors concluded that in patients with mild-to-moderately impaired hepatic function there is no need to adjust the dose. Cassidy et al (1999) applied univariate and multivariate regression analyses to study the influence of age, gender, body surface area and creatinine clearance on the main pharmacokinetic parameters of capecitabine and observed no clinically significant effects, the only statistically significant results being a higher AUC of intact capecitabine in females as compared to males ( $87 \%$ greater in females than in males). However, in patients with renal dysfunction and treated by capecitabine there was a significant increase in $5^{\prime}$ DFUR (Poole et al, 2002); this led to specifically recommend a dose modification of capecitabine for patients with very low creatinine clearance. More studies are needed to examine the possible influence of altered renal or hepatic function on the pharmacokinetics of oral fluoropyrimidines.

\section{PHARMACOKINETICS - PHARMACODYNAMICS RELATIONSHIPS}

For most oral fluoropyrimidines developed so far, PK-PD relationships were observed most often during phase I trials. For instance, Van Groeningen et al (2000) noted, for S-1, that among 5FU plasma concentrations measured during the 28 days of oral treatment, only the level just before drug intake in week 2 was significantly related to grade 3-4 diarrhoea. A confirmation of this interesting observation during phase II-III trials would be useful in order to identify a 5-FU threshold concentration at risk for toxicity with a possibility for dose adjustment during the treatment course. Steady-state plasma 5-FU concentrations were monitored during a phase I trial combining eniluracil-5FU treatment with radiotherapy (Humerickhouse et al, 1999). The authors noted that average drug concentrations correlated inversely with absolute neutrophil count nadirs but no correlation was observed with other toxicities. Muggia et al (1996), during the UFT timed-dose study, reported on marked toxicities (including life-threatening haematological toxicities) in patients exhibiting peak levels of 5-FU above $1 \mu \mathrm{g} \mathrm{ml}^{-1}$. Ho et al (2000) observed significant correlations between maximal plasma concentration or AUC $0-6 \mathrm{~h}$ of 5-FU and the occurrence of diarrhoea or nausea and vomiting during a phase I trial on UFT. This information could be potentially useful in order to perform an early pharmacokinetic-based dose adaptation of UFT. Since capecitabine generates 5-FU directly at cellular level, it can be anticipated that the variability in plasma pharmacokinetics of capecitabine and metabolite will be of little concern for pharmacodynamics. This opinion has been confirmed by clinical pharmacokinetic investigations (Reigner et al, 2001).

\section{PREDICTIVE MARKERS}

Whatever the oral fluoropyrimidine considered, all release 5-FU which is the final cytotoxic prodrug. Consequently, our clinical knowledge of the predictive markers for 5-FU-based treatment efficacy should apply to oral fluoropyrimidines. Ichikawa et al (2003) examined the relative tumoural expression of DPD and TS for advanced colorectal patients to be treated by UFT. The authors noted that no responding tumours had both high DPD and high TS and the response rate reached a satisfactory $75 \%$ in tumours with both low DPD and low TS. Capecitabine ultimately delivers 5-FU at the cellular level through the action of TP; in theory, the flux of 5FU production can be counterbalanced by a more or less marked opposite flux of 5-FU intracellular catabolism mediated by DPD. This view was recently confirmed by Tsukamoto et al (2001), who measured, in vitro, the enzyme kinetic parameters of each of the enzymes involved in the activation of capecitabine to 5-FU and elimination. The authors constructed a physiologically based pharmacokinetic model which revealed that the most important factors determining the selective production of 5-FU in tumour tissue after capecitabine administration were activation by TP, nonlinear elimination of 5-FU by DPD and the tumour blood flow rate. These data are in agreement with those reported by Ishikawa et al (1998) showing that the efficacy of capecitabine on a large panel of 24 human cancer xenografts was significantly correlated to the tumour TP/DPD ratio. Clinical confirmation of these experimental data would be of great interest. Overall, our current understanding of predictive markers for oral fluoropyrimidine treatment is limited and, with the exception of UFT, remains based on extrapolations from 5-FU clinical studies.

\section{PHARMACOMODULATION}

The first approach for pharmacologically modulating oral fluoropyrimidines is to incorporate a DPD inhibitor. This strategy allows 5-FU to be correctly absorbed, as is the case with the combination eniluracil-5FU (Hirata et al, 1999; Adjei et al, 2002). DPD inhibition also markedly prolongs 5-FU elimination half-life thus making the oral administration of fluoropyrimidines pharmacokinetically acceptable on the basis of two or three daily intakes. The second approach for a pharmacomodulation of oral fluoropyrimidines involves combination with leucovorin (LV). There is, however, an open debate about the clinical/pharmacological efficacy of LV supplementation during prolonged administration of 5-FU. Solutions to this dilemma are provided by 
experimental and clinical data. For instance, an interesting study by Codacci-Pisanelli et al (1995) examined the effects of adding LV on human cancer-xenografts in mice subcutaneously implanted with pellets releasing 5-FU over a period of 3 weeks. The authors clearly demonstrated that coadministration of LV did not enhance the therapeutic results. On the clinical side, a meta-analysis was previously reported showing the advantage of 5-FU plus LV over 5FU in terms of objective response rate (Advanced Colorectal Cancer Meta-analysis Project, 1992). However, all reports included in the analysis concerned clinical trials with rapid intravenous 5FU administrations. A clinical pharmacological study was specifically designed in gastric cancer patients to determine whether orally administered LV enhanced TS inhibition when added to UFT (Ichikura et al, 1996). The results showed that TS inhibition rate in tumours was significantly higher in the UFT-LV group than in the UFT group alone. However, the data were obtained following a short period of 3 consecutive days of treatment prior to surgery. Thus, it is not clear, as is the case with the current clinical protocols, whether prolonged exposure to UFT results in an equivalent TS inhibition rate with or without LV supplementation. On the other hand, Van Cutsem et al (2000) undertook a randomised phase II trial in advanced colorectal cancer patients aimed at selecting the most appropriate capecitabine regimen for testing in phase III studies. Two arms ( 2 weeks on/1 week off) differed by the presence or not of daily oral LV. Tumour responses were comparable between the two arms but more toxicity was seen in the LV-capecitabine arm. Thus, we do not dispose of strong experimental and clinical arguments to support LV supplementation during continuous treatment by oral fluoropyrimidines.

\section{REFERENCES}

Advanced Colorectal Cancer Meta-analysis Project (1992) Modulation of fluorouracil by leucovorin in patients with advanced colorectal cancer: evidence in terms of response rate. J Clin Oncol 10: 896-903

Adjei AA, Reid JM, Diasio RB, Sloan JA, Smith DA, Rubin J, Pitot HC, Alberts SR, Goldberg RM, Hanson LJ, Atherton P, Ames MM, Erlichman C (2002) Comparative pharmacokinetic study of continuous venous infusion fluorouracil and oral fluorouracil with eniluracil in patients with advanced solid tumours. J Clin Oncol 20: 1683-1691

Baker SD, Diasio RB, O'Reilly S, Sol Lucas V, Khor SP, Sartorius SE, Donehower RC, Grochow LB, Spector T, Hohneker JA, Rowinsky E (2000) Phase I and pharmacologic study of oral fluorouracil on a chronic daily schedule in combination with the dihydropyrimidine dehydrogenase inactivator eniluracil. J Clin Oncol 18: 915-926

Cassidy J, Twelves C, Cameron D, Steward W, O’Byrne K, Jodrell D, Banken L, Goggin T, Jones D, Roos B, Bush E, Weidekamm E, Reigner B (1999) Bioequivalence of two tablet formulations of capecitabine and exploration of age, gender, body surface area, and creatinine clearance as factors influencing systemic exposure in cancer patients. Cancer Chemother Pharmacol 44: $453-460$

Codacci-Pisanelli G, Van der Wilt CL, Pinedo HM, Franchi F, Noordhuiso P, Braakhuis Bj, van Laar JA, Peters GJ (1995) Antitumour activity toxicity and inhibition of thymidylate synthase of prolonged administration of 5-fluorouracil in mice. Eur J Cancer 31: 1517-1525

Damle B, Ravandi F, Kaul S, Sonnichsen D, Ferreira I, Brooks D, Stewart D, Alberts D, Pazdur R (2001) Effect of food on the oral bioavailability of UFT and leucovorin in cancer patients. Clin Cancer Res 7: $517-523$

Diasio RB (1999) Improving fluorouracil chemotherapy with novel orally administered fluoropyrimidines. Drugs 58: 119-126

Endo M, Miwa M, Eda H, Ura M, Tanimura H, Ishikawa T, Miyazaki-Nose T, Hattori K, Shimma N, Yamada-Okabe H, Ishitsuka H (2003) Augmentation of the antitumour activity of capecitabine by a tumour selective dihydropyrimidine dehydrogenase inhibitor, RO0094889. Int J Cancer 106: 799-805

Grem JL (1996) 5-Fluoropyrimidines. In Cancer Chemotherapy and Biotherapy, Chabner BA, Longo DL (eds) pp 149-212, Philadelphia, PA: Lipppincott-Raven
Apart from DPD and LV handling which can apply to most oral fluoropyrimidines, there are more specifically product-oriented strategies which can be of potential interest for the pharmacomodulation of these drugs. This is particularly true for UFT and capecitabine. Reports have been published of attempts to modulate the activity of $5^{\prime}$ DFUR, and thus that of capecitabine, through the upregulation of TP. Cytotoxic agents, particularly taxanes and irradiation, are able to increase TP activity and result in synergistic interaction with capecitabine on experimental models in vitro and in vivo (Sawada et al, 1998, 1999). However, the exact mechanistic cause of the TP modulation by the cytotoxics has not been clearly elucidated and indirect effects via the upregulation of TNF $\alpha$ have been advocated (Sawada et al, 1998, 1999). The preclinical findings of supra-additive antitumour effects of combining taxanes and capecitabine have been followed by promising clinical results in breast cancer treatment with a protocol based on a capecitabinedocetaxel combination (O'Shaughnessy et al, 2002). Recent findings suggest that cytochrome P-4502A6 is the major enzyme responsible for the bioactivation process of ftorafur (Ikeda et al, 2000). Further studies would be of benefit to explore at the tumour level a possible activation of ftorafur into 5-FU via CYP2A6 and the possible modulation of this enzymatic pathway.

These possibilities to achieve pharmacomodulation of oral fluoropyrimidines, notably for UFT and capecitabine, opens up the prospect of establishing significant novel treatment protocols based on drug combinations. This increasing number of oral formulations for several cytotoxic drugs may well make it possible to provide 'all oral' treatments with advantages for patients in terms of quality of life.
Hattori K, Kohchi Y, Oikawa N, Suda H, Ura M, Ishikawa T, Miwa M, Endoh $M$, Eda $H$, Tnaimura $H$, Kawashima A, Horii I, Ishitsuka $H$ Shimma N (2003) Design and synthesis of the tumour-activated prodrug of dihydropyrimidine dehydrogenase (DPD) inhibitor, RO0094889 for combination therapy with capecitabine. Bioorg Med Chem Lett 13: $867-872$

Hirata K, Horikoshi N, Aiba K, Okazaki M, Denno R, Sasaki K, Nakano Y, Ishizuka H, Yamada Y, Uno S, Taguchi T, Shirasaka T (1999) Pharmacokinetic study of S-1, a novel oral fluorouracil antitumour drug. Clin Cancer Res 5: 2000-2005

Ho DH, Pazdur R, Covington W, Brown N, Huo YY, Lassere Y, Kuritani J (1998) Comparison of 5-fluorouracil pharmacokinetics in patients receiving continuous 5-fluorouracil infusion and oral uracil plus $N_{1}$-(2'-tetrahydrofuryl)-5-fluorouracil. Clin Cancer Res 4: 2085-2088

Ho DH, Covington W, Brown N, Lin SN, Pazdur R, Huo YY, Creaven PJ, Rustum YM, Meropol NJ, Lassere Y, Kuritani J, Hayadawa T (2000) Oral uracil and ftorafur plus leucovorin: pharmacokinetics and toxicity in patients with metastatic cancer. Cancer Chemother Pharmacol 46: $351-356$

Humerickhouse RA, Dolan ME, Haraf DJ, Brockstein B, Stenson K, Kies M, Sulzen L, Ratain MJ, Vokes EE (1999) Phase I study of eniluracil, a dihydropyrimidine dehydrogenase inactivator, and oral 5-fluorouracil with radiation therapy in patients with recurrent or advanced head and neck cancer. Clin Cancer Res 5: 291-298

Ichikura T, Tomimatsu S, Okusa Y, Yahara T, Uefuji K, Tamakuma S (1996) Thymidylate synthase inhibition by an oral regimen consisting of tegafur-uracil (UFT) and low-dose leucovorin for patients with gastric cancer. Cancer Chemother Pharmacol 38: 401-405

Ikeda K, Yoshisue K, Matsushima E, Nagayama S, Kobayashi K, Tyson CA, Chiba K, Kawaguchi Y (2000) Bioactivation of tegafur to 5-fluorouracil is catalyzed by cytochrome $P-4502 \mathrm{~A} 6$ in human liver microsomes in vitro. Clin Cancer Res 6: 4409-4415

Ishikawa T, Sekiguchi F, Fukase Y, Sawada N, Ishitsuka H (1998) Positive correlation between the efficacy of capecitabine and doxifluridine and the ratio of thymidine phosphorylase to dihydropyrimidine dehydrogenase activities in tumours in human cancer xenografts. Cancer Res 58: $685-690$ 
Ichikawa W, Uetake H, Shirota Y, Yamada H, Nishi N, Nihei Z, Sugihara K, Hirayama R (2003) Combination of dihydropyrimidine dehydrogenase and thymidylate synthase gene expressions in primary tumours as predictive parameters for the efficacy of fluoropyrimidine-based chemotherapy for metastatic colorectal cancer. Clin Cancer Res 9: $786-791$

Mader RM, Schrolnberger C, Rizovski B, Brunner M, Wenzel C, Locker G, Fichler HG, Mueller M, Steger GG (2003) Penetration of capecitabine and its metabolites into malignant and healthy tissues of patients with advanced breast cancer. B J Cancer 88: $782-787$

Meropol NJ, Sonnichsen DS, Birkhofer M, Ferreira I, Noel D (1999) Bioavailability and phase II study of oral UFT plus leucovorin in patients with relapsed or refractory colorectal cancer. Cancer Chemother Pharmacol 43: $221-226$

Muggia FM, Wu X, Spicer D, Groshen S, Jeffers S, Leichman G, Leichman L, Chan KK (1996) Phase I and pharmacokinetic study of oral UFT, a combination of the 5-fluorouracil prodrug tegafur and uracil. Clin Cancer Res 2: $1461-1467$

Nemunaitis J, Eager R, Twaddell T, Corey A, Sekar K, Tkaczuk K, Thompson J, Hoff PM, Pazdur R (2000) Phase I assessment of the pharmacokinetics, metabolism, and safety of emitefur in patients with refractory solid tumours. J Clin Oncol 18: $3423-3434$

O'Shaughnessy J, Miles D, Vukelja S, Moiseyenko V, Ayoub JP, Cervantes G, Fumoleau P, Jones S, Lui WY, Mauriac L, Twelves C, Van Hazel G, Verma S, Leonard R (2002) Superior survival with capecitabine plus docetaxel combination therapy in anthracycline-pretreated patients with advanced breast cancer: phase III trial results. J Clin Oncol 20: $2812-2823$

Peters GJ, Noordhuis P, Van Kuilenburg AB, Schornagel JH, Gall H, Turner SL, Swart MS, Voom D, Van Gennip AH, Wanders J, Holwerda U, Smid K, Giaccone G, Fumoleau P, Van Groeningen CJ (2003) Pharmacokinetics of $\mathrm{S}-1$, an oral formulation of ftorafur, oxonic acid and 5-chloro-2,4dihydroxypyridine (molar ratio $1: 0.4: 1$ ) in patients with solid tumours. Cancer Chemother Pharmacol 52: 1-12

Petit E, Milano G, Levi F, Thyss A, Bailleul F, Schneider M (1988) Circadian rhythm-varying plasma concentration of 5-fluorouracil during a five day continuous venous infusion at a constant rate in cancer patients. Cancer Res 48: 1976-1979

Pivot X, Chamorey E, Guardiola E, Magn N, Thyss A, Otto J, Giroux B, Mouri Z, Schneideer M, Milano G (2003) Phase I and pharmacokinetic study of the association of capecitabine-cisplatin in head and neck cancer patients. Ann Oncol 14: 1578-1586

Poole C, Gardiner J, Twelves C, Johnston P, Harper P, Cassidy J, Monhouse J, Banken L, Weidekamm E, Reigner B (2002) Effect of renal impairment on the pharmacokinetics and tolerability of capecitabine (Xeloda) in cancer patients. Cancer Chemother Pharmacol 49: 225-234

Reigner B, Verweij J, Dirix L, Cassidy J, Twelves C, Allman D, Weidekamm E, Roos B, Banken L, Utoh M, Osterwalder B (1998) Effect of food on the pharmacokinetics of capecitabine and its metabolites following oral administration in cancer patients. Clin Cancer Res 4: 941-948

Reigner B, Blesch K, Weidekamm E (2001) Clinical pharmacokinetics of capecitabine. Clin Pharmacokinet 40: 85-104

Sadahiro S, Suzuki T, Kameya T, Iwase H, Tajima T, Makuuchi H (2001) A pharmacological study of the weekday-on/weekend-off oral UFT schedule in colorectal cancer patients. Cancer Chemother Pharmacol 47: $457-460$

Sawada N, Ishikawa T, Fukase Y, Nishida M, Yoshikubo T, Ishitsuka H. (1998) Induction of thymidine phosphorylase activity and enhancement of capecitabine efficacy by taxol/taxotere in human cancer xenografts. Clin Cancer Res 4: 1013-1019

Sawada N, Ishikawa T, Sekiguchi F, Tanaka Y, Ishitsuka H (1999) X-ray irradiation induces thymidine phosphorylase and enhances the efficacy of capecitabine (xeloda) in human cancer xenografts. Clin Cancer Res 5: $2948-2953$

Shepard DR, Mani S, Kastrissios H, Learned-Coughlin S, Smith D, Ertel P, Magnum S, Janish L, Fleming GF, Schilsky RL, Ratain MJ (2002) Estimation of the effect of food on the disposition of oral 5-fluorouracil in combination with eniluracil. Cancer Chemother Pharmacol 49: $398-402$

Shimasaka T, Shimamoto Y, Ohshimo H, Yamaguchi M, Kato T, Yonekura K (1996) Development of a novel form of an oral 5-fluorouracil derivative (S-1) directed to the potentiation of the tumour selective cytotoxicity of 5-fluorouracil by two biochemical modulators. Anticancer Drugs 7: $548-557$

Schüller J, Cassidy J, Dumont E, Roos B, Durston S, Banken L, Utoh M, Mori K, Weidekamm E, Reigner B (2000) Preferential activation of capecitabine in tumour following oral administration to colorectal cancer patients. Cancer Chemother Pharmacol 45: 291-297

Tachibana M, Yasuda N, Yoshimatsu M, Nishimura H, Mizukoshi O (1987) UFT for head and neck cancers: its tissue concentrations and effects on lymphocyte subpopulations. Cancer Chemother Pharmacol 19: $65-68$

Tatsumi K, Yamauchi T, Kiyono D, Kishi K, Yanagihara Y, Imaoka T, Kawaguchi T, Kubo M (1993) 3-Cyano-2,6-dihydroxypyridine (CNDP), a new potent inhibitor of dihydrouracil dehydrogenase. J Biochem 114: 912-918

Tsukamoto Y, Kato Y, Ura M, Horii I, Ishituska H, Kusuhara H, Sugiyama Y (2001) A physiologically-based pharmacokinetic analysis of capecitabine, a triple prodrug of 5-FU, in humans: the mechanism for tumourselective accumulation of 5-FU. Pharm Res 18: 1190-1202

Twelves C, Glynne-Jones R, Cassidy J, Schüller J, Goggin T, Roos B, Banken L, Utoh M, Weidekamm E, Reigner B (1999) Effect of hepatic dysfunction due to liver metastases on the pharmacokinetics of capecitabine and its metabolites. Clin Cancer Res 5: 1696-1702

Urien S, Fumoleau P, Campone M, Kerbrat P, Bonneterre J, Fargeot P, Deporte-Fety R (2003) Modelling of ftorafur and 5-fluorouracil pharmacokinetics following oral UFT administration. A population study in 30 patients with advanced breast cancer. Cancer Chemother Pharmacol 52: 99-107

Van Cutsem E, Findlay M, Osterwalder B, Kocha W, Dalley D, Pazdur R, Cassidy J, Dirix L, Twelves C, Allman D, Seitz JF, Schölmerich J, Burger HU, Verweij J (2000) Capecitabine, an oral fluoropyrimidine carbamate with substantial activity in advanced colorectal cancer: results of a randomized phase II study. J Clin Oncol 18: 1337-1345

Van Groeningen CJ, Peters GJ, Schornagel JH, Gall H, Noordhuis P, de Vries MJ, Turner SL, Swart MS, Pinedo HM, Hanauske AR, Giaccone G (2000) Phase I clinical and pharmacokinetic study of oral S-1 in patients with advanced solid tumours. J Clin Oncol 18: 2772-2779 